\title{
Precise charged particle timing with the PICOSEC detector
}

\section{Bortfeldt, J.}

2019

Bortfeldt , J Brunbauer , F M , David , C , Desforge , D , Fanourakis , G , Franchi , J , Gallinaro , M , Garcia , F , Giomataris , I , Gustavsson , T , Guyot , C , Iguaz , F J , Kebbiri , M , Kordas , K, Legou , P , Liu , J , Lupberger , M , Maillard, O , Manthos , I , Muller , H , Niaouris , V, Oliveri , E, Papaevangelou , T , Paraschou , K, Pomorski , M , Qi , B , Resnati , F , Ropelewski , L, Sampsonidis , D , Schneider , T , Schwemling , P , Sohl , L, van Stenis , M , Thuiner , P , Tsipolitis , Y, Tzamarias, S E , Veenhof , R , Wang , X, White , S , Zhang , Z \& Zhou , Y 2019 , ' Precise charged particle timing with the PICOSEC detector ' , AIP Conference Proceedings , vol. 2075 , no. 080009 , 080009 . https://doi.org/10.1063/1.5091210

http://hdl.handle.net/10138/312215

https://doi.org/10.1063/1.5091210

cc_by

acceptedVersion

Downloaded from Helda, University of Helsinki institutional repository.

This is an electronic reprint of the original article.

This reprint may differ from the original in pagination and typographic detail.

Please cite the original version. 


\title{
Precise Charged ParticleTiming with the PICOSEC Detector
}

\author{
J. Bortfeldt'2, F. Brunbauer2 , C. David², D. Desforge' ${ }^{1}$, G. Fanourakis ${ }^{5}$, J. Franchi², \\ M. Gallinaro ${ }^{7}$, F. García ${ }^{11}$, I. Giomataris' ${ }^{1}$, T. Gustavsson ${ }^{9}$, C. Guyot ${ }^{1}$, F.J . Iguaz ${ }^{1}$,

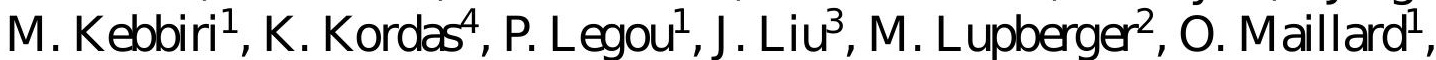 \\ I. Manthos ${ }^{4, a}$, H. Müller' ${ }^{2}$, V. Niaouris ${ }^{4}$, E. Oliveri ${ }^{2}$, T. Papaevangelou' ${ }^{1}$, \\ K. Paraschou ${ }^{4}$, M. Pomorski ${ }^{10}$, B. $\mathrm{Qi}^{3}$, F. Resnati ${ }^{2}$, L. Ropelewski ${ }^{2}$, \\ D. Sampsonidis ${ }^{4}$, T. Schneider ${ }^{2}$, P. Schwemling ${ }^{1}$, L. Sohl ${ }^{1}$, M. van Stenis ${ }^{2}$,

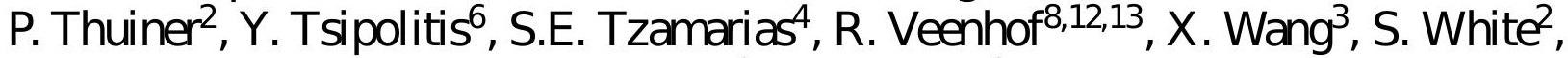 \\ Z. Zhang ${ }^{3}$ and Y. Zhou ${ }^{3}$
}

\author{
${ }^{1}$ IRFU, CEA, Universite Paris-Saclay, F-91191 Gif-sur-Yvette, France \\ 2European Organization for Nuclear Research (CERN), CH-1211 Geneve 23, Switzerland \\ ${ }^{3}$ State Key Laboratory of Particle Detection and Electronics, Uni versity of Science and Technol ogy of China, Hefei \\ CN-230026, China \\ ${ }^{4}$ Department of Physics, Aristotle University of Thessal oniki, University Campus, GR-54124, Thessal oniki, Greece \\ 5 Institute of Nuclear and Particle Physics, NCSR Demokritos, GR-15341 Agia Paraskevi, Attiki, Greece \\ ${ }^{6}$ National Technical University of Athens, Athens, Greece \\ ${ }^{7}$ Laboratório de Instrumentacão e Física Experimental de Partículas, Lisbon, Portugal \\ ${ }^{8}$ RD51 collaboration, European Organization for Nuclear Research (CERN), CH-1211 Geneve 23, Switzerland \\ ${ }^{9}$ LIDYL, CEA, CNRS, Universit Paris-Saclay, F-91191 Gif-sur-Yvette, France \\ ${ }^{10}$ CEA-LIST, Diamond Sensors Laboratory, CEA Saclay, F-91191 Gif-sur-Yvette, France \\ ${ }^{11}$ Helsinki Institute of Physics, University of Helsinki, FI-00014 Helsinki, Finland \\ ${ }^{12}$ National Research Nuclear University MEPhl, Kashirskoe Highway 31, Moscow, Russia \\ ${ }^{13}$ Department of Physics, Uludag University, TR-16059 Bursa, Turkey \\ a)Corresponding author: ioannis.manthos@cem.ch
}

\begin{abstract}
The experimental requirements in near future accelerators (eg. High Luminosity-LHC) has stimul ated intense interest in devel opment of detectors with high precision timing capabilities. With this as a goal, a new detection concept called PICOSEC, which is based to a "two-stage" MicroMegas detector coupled to a Cherenkov radiator equipped with a photocathode has been developed. Resul ts obtained with this new detector yield a time resolution of $24 \mathrm{ps}$ for $150 \mathrm{GeV}$ muons and $76 \mathrm{ps}$ for single photoelectrons. In this paper we will report on the performance of the PICOSEC in test beams, as well as simulation studies and modelling of its timing characteristics.
\end{abstract}

\section{INTRODUCTION}

It is very well known that in order to take advantage of the High Luminosity $(\mathrm{HL})$ of the Large Hadron Collider (LHC) we need to have the ability to tag events which are promising to carry signatures of new physics. The experimental signature of such events (eg. forward energetic jets) are di cult to be identified in the very dense topology of the

140 interactions per beam crossing in the HL-LHC. The LHC experiments are forced to use precise timing detectors to expl ore the possi bilities the accelerators o er. Today, there are avai lable detectors that provide timing of a few ps per Minimum I onising Particle (MIP), par example the Micro Channel Plate (MCP) detector. However, since the 


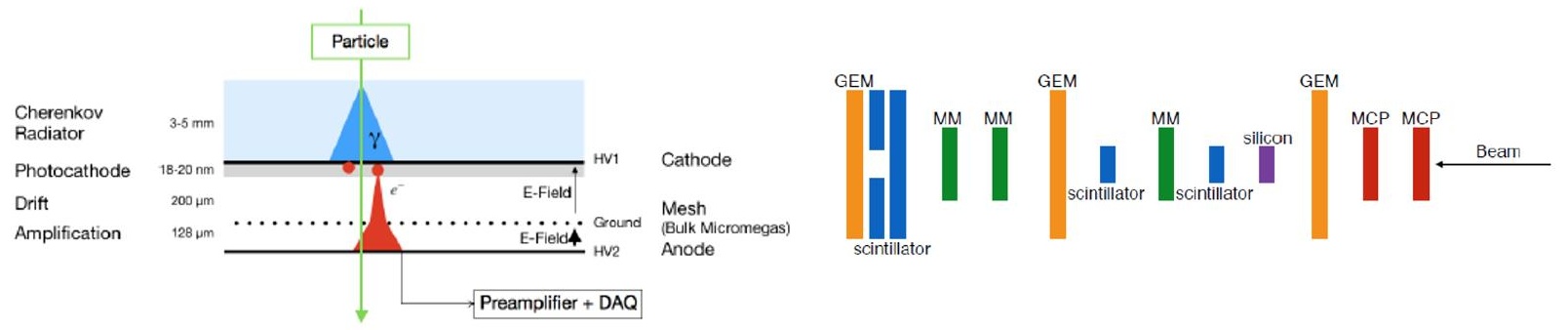

FIGURE 1: The layout of the PICOSEC MicroMegas detector [3] (left). Sketch of the experimental setup in the $150 \mathrm{GeV}$ muon test beam (right).

hermetic approach at the LHC and future accelerator experiments requires large area coverage, it is only natural to investigate more economical solutions, such as Micro-Patern Gas and Silicon structures, to o er such timing capabilities. However, since the necessary timeresolution for pileup mitigation is of the order of 20-30 ps, both technologies require significant modifications to reach the desired performance. Eventually, there is need for large area detectors, resistant to radiation damage with 10 ps timing capabilities, that will also find applications in various domains beyond Particle Physics experiments. Photon's energy/speed measurements and correlations for Cosmology, optical tracking for charged particles, 4D tracking in the future accelerators etc. In this paper it is reported the performance of the very successful technology of MicroMegas (MM) detectors, for precise timing purposes. Focus is given to the test beam cal ibration runs, data analysis and the physics of this detector.

\section{THE PICOSEC DETECTOR CONCEPT}

There is a seminal paper by Sauli [1] that explicitly proves that proportional gaseous detectors, due to the statistics of the ionization can hardly exceed the ns-level for the time resolution per particle ( 6 ns for typical chamber dimensions). Recently, the RD-51 PICOSEC collaboration developed a detection technique [2] and published work [3, 4] demonstrating that a special MicroMegas detector can reach a timing resolution better than $25 \mathrm{ps}$. In this detector (hereafter PICOSEC-MM) the drift region has been reduced to less than $200 \mu \mathrm{m}$ in order to practically mini mize the

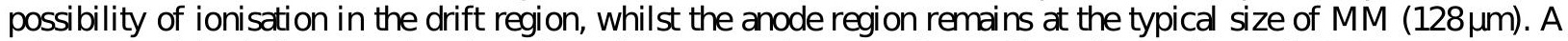
primary particle, before entering the drift region, passes through a $3 \mathrm{~mm}$ Cherenkov radiator of $\mathrm{MgF}_{2}$ and the produced photons generate photoelectrons on a photocathode, placed just below the radiator, in contact with the gas volume of the drift region, as on Fig. 1 (left). The photocathode has been deposited on a thin film of semitransparent $\mathrm{Cr}$ which is used to provide conductivity to the cathode. The photoelectrons are produced al most synchronously and start avalanches in the drift region due to the high electric field produced by modest vol tage di erences (of the order of $400 \mathrm{~V}$ ). The preamplification aval anche in the drift region is shown schematically in Fig. 1 (left).

\section{Muon Test Beam Results}

ThePICOSEC -MM detector has been tested extensively at the CERN SPS H4 secondary beamline. The experimental setup [3], shown in Fig. 1 (right) comprise scintillation counters for triggering, GEM detectors for tracking the muons of the beam, MCP detectors to provide time reference with a resolution better than $6 \mathrm{ps}$ and the under test PICOSECMM detectors, with COMPASS gas filling ( $\left.\mathrm{Ne}+10 \% \mathrm{C}_{2} \mathrm{H}_{6}+10 \% \mathrm{CF}_{4}\right)$ at 1 bar. The PICOSEC-MM signal was amplified by a CIVIDEC amplifier ( $2 \mathrm{GHz}, 40 \mathrm{~dB}$ ) and the waveform was digitized by a $2.5 \mathrm{GHz}$ oscilloscope at a rate of 20 GSamples/s (i.e one sample every 50 ps). A typical pulse of the PICOSEC-MM responding to a muon is presented in Fig. 2 (left). The very fast component with a rise time of $500 \mathrm{ps}$ and a duration of the order of $\mathrm{ns}$ is due to the induction of current on the anode by the fast moving electrons, whilst the slow component due to the slow moving ions is extended up to several hundreds $\mathrm{ns}$. The collected digitised waveforms were analysed o ine. Standard analysis procedure was applied to determine the beginning of the pulse, the el ectron peak (hereafter e-peak) amplitude, the end of the epeak and the arrival time of the pulse. A crucial point in this analysis was to estimate accurately the pulse characteristics, especially the arrival time, even if there is a non-negl igible noise contribution to 

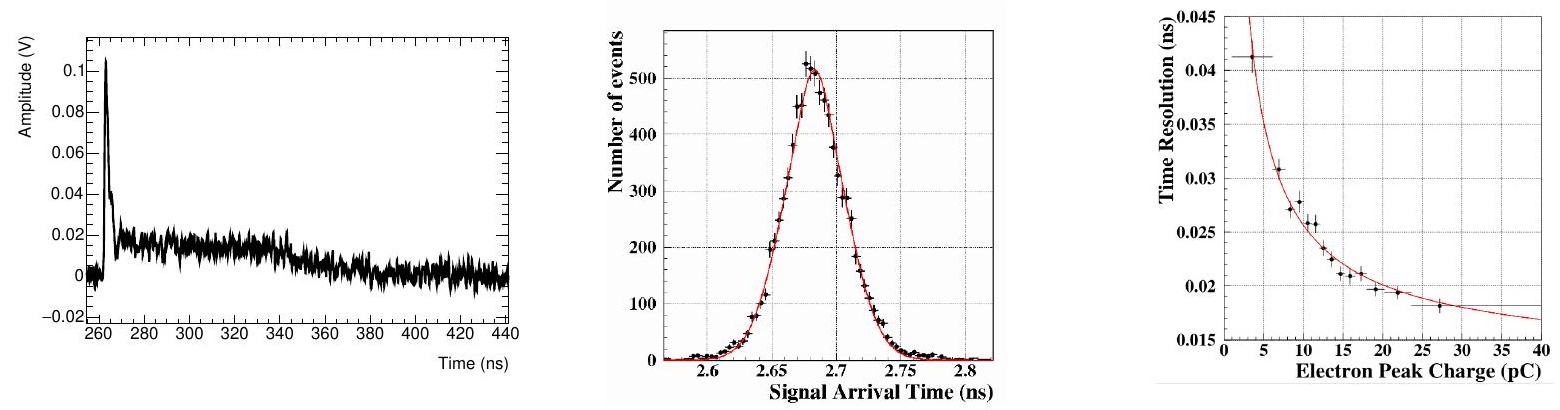

FIGURE 2: A typical pulse produced by the PICOSEC-MM detector (left). SAT distribution for $150 \mathrm{GeV}$ muons, and the fit with a two Gaussian function resulting to $24 \mathrm{ps}$ timing resolution, for anode and drift vol tage of $275 \mathrm{~V}$ and 475V respectively (center). The dependence of the resolution as a function of the e-peak pul se's charge (right).
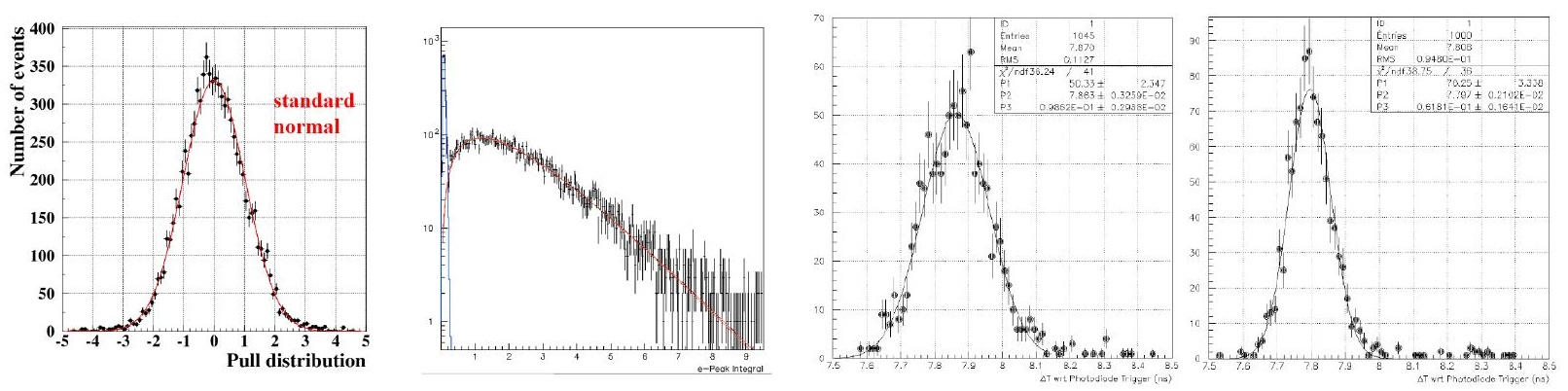

FIGURE 3: The pull distribution of thetiming resolution dependence on thee-peak charge on an event by event basis (left). Distribution of the e-peak charge collected by the detector response to a single photoelectron. This distribution was parametrized by a Polya function (red), whilst the noise (blue) is parametrized and rejected (central left). SAT distribution for two e-peak charge regions: The first corresponds to low charge values while the second to high charge values. Mean val ues are di erent, as larger pulses are coming earlier and with better timing resolution (central right and right plot respectively).

the digitised waveform. It was found that by processing the signal using filtering al gorithms [5] the noise contribution was reduced but the very fast e-peak leading edge was a ected. A processing anal ysis al gorithm was devel oped, by fitting the leading edge of the e-peak using a logistic function, which o ers the desired accuracy to estimate theSignal Arrival Time (SAT), which was defined as the point that the fitted leading edge was crossing a threshold of the $20 \%$ of the peak maximum (i.e. the standard technique of the Constant Fraction Discrimination (CDF)). Results achieved with $150 \mathrm{GeV}$ muon beam with $275 \mathrm{~V}$ anode and $475 \mathrm{~V}$ drift voltage settings are presented in Fig. 2 (center) where the distribution of the SAT with respect to the MCP time reference is shown. The distribution is fitted by the sum of two Gaussians and the combined Root Mean Square (RMS) was found to be 24:0 0:3 ps which is the timing resolution per MIP of the detector on these vol tage settings. In the right plot of Fig. 2 the RMS versus e-peak charge is shown. These distributions (i.e SAT distributions in narrow bins of the epeak charge) wherefound to be Gaussian, however as shown in the right pl ot of Fig. 2 the PICOSEC-MM resolution (RMS) depends on the epeak charge. This dependence is also the reason that the distribution at the central plot of Fig. 2, corresponding to a wide spectrum of e-peak charge, deviates from the Gaussian shape. Using the parametrization of the resolution on the e-peak charge on event by event basis, the pull distribution is determined as shown in the left of Fig. 3. Thefact that the pull distribution is, in a very good approximation, nomal Gaussian, supports the argument described above.

\section{Single Photoelectron Test Results}

The PICOSEC-MM signal, generated by a muon, is the sum of the detectors response to each of the produced photoelectrons. To study the PICOSEC-MM timing characteristics, the detector response to a single photoelectron was also studied in special runs in Ref. [3]. The photocathode was illuminated using a femptosecond laser beam with very 

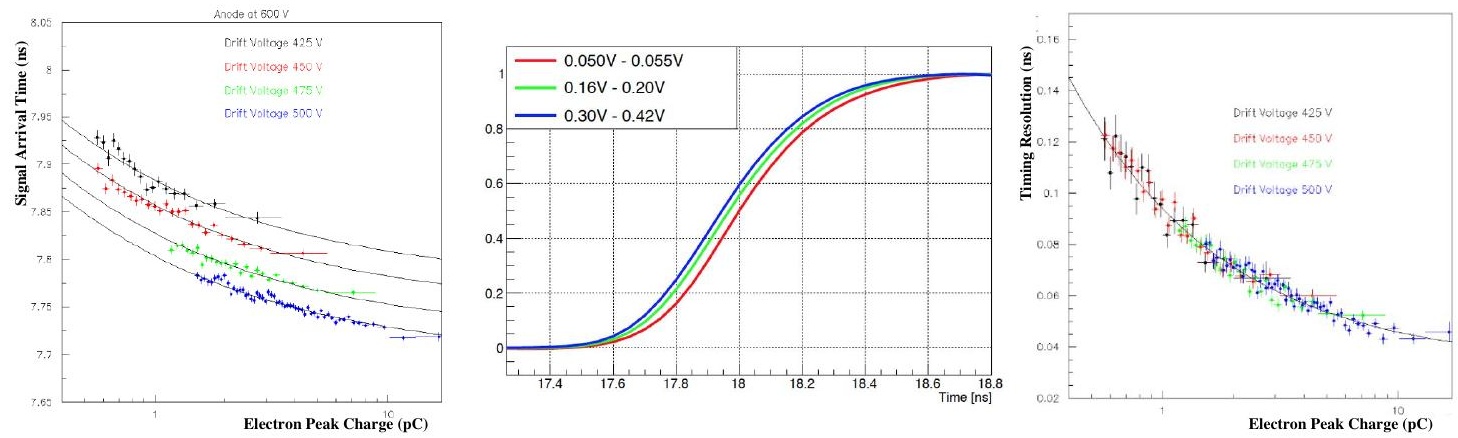

FIGURE 4: SAT as a function of theepeak size for several drift voltages and anode at $600 \mathrm{~V}$ (left). Average of pulses corresponding to di erent charge regions normalized to the unit. The shape does not change but higher pulses arrive earlier (center). Thetiming resol ution dependence on the e peak size, for constant anodefield $600 \mathrm{~V}$ and di erent drift voltages as mentioned in the plot (right).

low intensity, so as to produce a single photoel ectron. The laser beam was split, a portion was illuminating a precise photodiode, which provides a time reference, while the other part illuminates the PICOSEC-MM. Both signals were digitised by a very fast oscilloscope. The distribution of the e-peak charge corresponding to a single photoel ectron is presented in Fig. 3 (central left). The experimental distribution has been fitted by a Polya function plus a distribution accounting for the background contribution which has been modelled using out of time events in Ref. [6]. The SAT distribution (that is the time di erence of the PICOSEC-MM pulse arrival time and the reference photodiode pulse timing) for waveforms with e-peak charges in two regions, is shown in center right and right plots of Fig. 3. The first corresponds to low charge val ues (1.14 - $1.69 \mathrm{pC})$ and the second to high charge values ( $7.96-10.40 \mathrm{pC})$. As shown in these plots, both the mean values and the RMSs of the two distributions are di erent, signifying that larger pulses are coming earlier and with better timing resolution than smaller pulses. By studying the SAT distribution in bins of the e-peak charge, the dependence of the mean arrival time and the timing resolution on the e-peak size was studied. Specifically the mean arrival time is shown as a function of the epeak pulse size for several drift voltages in Fig. 4 (left). The mean SAT dependence on the e-peak size found to follow a functional form of Eq. (1).

$$
g(x ; \quad ; b ; w)=+\frac{b}{x^{w}}
$$

The power law parameter $\mathrm{w}$, was found to beindependent of the drift vol tage settings but the constant terma found to change with the drift voltage. The latter is easy to explain as the drift velocity depends on the drift voltage. However when the constant term has been subtracted all the points are following the same curve, independently of the drift voltage. Many tests [7] have proven that the dependence of the arrival time on the e-peak size is not a systematic error due to the timing method. As shown in Fig. 4 (center), the average pulse shapes corresponding to di erent epeak charge regions retain a constant shape but the higher pulses are arriving earlier, demonstrating that this "time walk" is related to a shift of the whole pul se for reasons that have nothing to do with the experimental procedure, but resul ted by the pulse formation dynamics. In the right of Fig. 4, the resolution's dependence on the size of the pulse (measured as the charge of the electron pul se) is shown. It is apparent that the resolution depends on the pul se size, but this dependence is the same for all drift voltages. Results presented until now are related with data taken with $600 \mathrm{~V}$ voltagein the anode. Same dependences for SAT distributions and timing resolution are presented, whereevery colour corresponds to di erent anode voltage (left and central left of Fig. 5). The fact is that the avalanches at the anode region do not a ect the timing characteristics, but just amplify the signal.

\section{SIMULATION STUDIES}

In order to investigate these finding a detai led simulation was developed, based on Garfield++ [8]. In Ref. [9, 10], a technique which extracts the response of the el ectronics to a single amplification aval anche by comparing Garfield++ simulation with real data, is described in detai l. Using the estimated responsefunction for each electron arriving at the ampl ification region and assuming a linear response of the electronics, the simulation produces waveforms, including 
FIGURE 5: TheMean SAT correction versus e-peak chargeand timeresolution versus e-peak chargewith COMPASS gas mixture. Red corresponds to anode voltage $450 \mathrm{~V}$, green to $475 \mathrm{~V}$, blue to $650 \mathrm{~V}$ and magenta to $525 \mathrm{~V}$ (left and central left). The experimental average waveform (black points) for events with e-peak charge above $15 \mathrm{pC}$. This region was chosen to prevent the SAT dependence a ect the result The fit result is shown in the red line of the pl ot. The dashed line corresponds to the right limit of the fit region (central right). Distribution of the simulated e-peak charge (blue), scal ed with a factor $\mathrm{G}$, such that the distribution matches the experimental one (red). Anode vol tage is $450 \mathrm{~V}$ and drift voltage is $400 \mathrm{~V}$. Scaling factor for this case is $\mathrm{G}=27: 8$ (right).

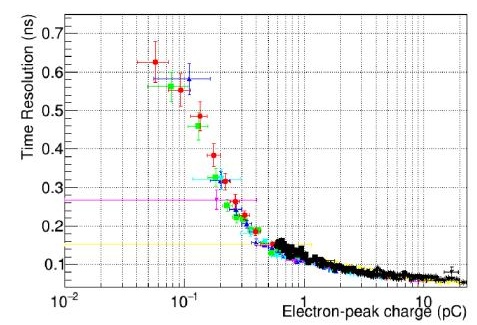

FIGURE 6: Mean SAT as a function of the e-peak charge (left). Time resolution as a function of the epeak charge (center). In both figures, black points correspond to experimental data while coloured correspond to simulated points with an anode voltage of $450 \mathrm{~V}$ and for drift voltages of (red) 300V, (green) 325V, (blue) 350V, (cyan) 375V, (magenta) $400 \mathrm{~V}$ and (yellow) 425V. Dependence of the mean transmission times with respect to the length of the aval anche (right).

a $2.5 \mathrm{mV}$ RMS uncorrelated noise. As an example, in Fig. 5 (central right) the average of real (black) and simulated (red) waveforms for epeaks with charges around $15 \mathrm{pC}$ is presented. The chamber was operated with $450 \mathrm{~V}$ and 425V anode and drift voltages, respectively. It should be noted that the prediction curve (in red) does not include the ion pulse. As a by-product of comparing distributions of simulated and real data, the Penning transfer rate of the COMPASS gas filling was also estimated to be around $50 \%$. An other example demonstrating the successful simulation of the PICOSEC-MM response is shown on Fig. 5 (right), where the epeak charge distribution for real data and simulated pulses are compared. The simulated pul ses were digitised and anal ysed exactly the same way as the real waveforms. The resolution and theSAT were determined in bins of the e-peak charge. The dependence of the timing characteristics of the simulated waveform on the e-peak size is shown in left and center Fig. 6 in comparison to the respecting results found in the real data. The agreement between the experimental data and the simulation is apparent.

\section{PHENOMENOLOGICAL MODELLING}

Having established an agreement between Garfield++ prediction and the data, the next step was to identify the microscopic physical parameters which correspond to the experimentally observed SAT. In Ref. [9] was found that the microscopic parameter which corresponds to the SAT is the average of the times of those of the preamplification electrons passing through the mesh when they pass the mesh. This average time was found to have the same statistical properties as SAT, i.e the same mean and RMS val ues in every bin of the epeak charge. The statistical properties of 
FIGURE 7: Dependence of the mean transmission times with respect to the number of preamplification aval anche electrons (left). Dependence of the spread of thetransmission times with respect to thelength of the aval anche (center). Spread of the transmission times depending on the number of preamplification aval anche electrons (right).

this microscopic variable are determined by the transmission time of the photoelectron from its emission to the first ionisation point and on the ective drift time of the aval anche. The dependence of the mean transmission times with respect to the length of the avalanche is shown in Fig. 6 (right). Both the photoelectron (red) and the aval anche (blue) mean transmission times have linear relation with the length of the avalanche, whose sum (black) is not constant but also depends linearly on the aval anche length. The dependence of the mean time when passing through the mesh (green) di ers only by a constant val ue. This dependencehints that the photoel ectron and the aval anche drift with different velocities. Fig. 7 (left) shows (with the same colour code) the dependence of the above mean transmission times with respect to the, experimentally observable, number of preamplification avalanche electrons. This shows that there is a "time wal k" e ect emerging in the microscopic simulation with Garfield++. The central plot of Fig. 7 presents the dependence of the spread of the transmission times with respect to the length of the aval anche, with the same color code. The spread of the photoelectron's transmission time increases with larger drift paths, while the spread of the aval anche's transmission time is saturated at a constant val ue. Therefore, the sooner the photoelectron ionizes for the first time, the better the time resolution. Fig. 7 (right) shows the spread of the transmission times depending on the number of preamplification avalanche electrons, with the same col or code. Notice that the transmission time spreads of the photoelectron and the avalanche are larger than the total time spread. This is due to the fact that the photoelectron and aval anche transmissi on times are heavily correlated.

\section{ACKNOWLEDGEMENTS}

We acknowledge the financial support of the RD51 collaboration, in the framework of RD51 common projects, the Cross-Disciplinary Program on Instrumentation and Detection of CEA, the French Alternative Energies and Atomic Energy Commission; and theFundamental Research Funds for the Central Universities of China. J . Bortfel dt acknowledges the support from the COFUND-FP-CERN-2014 program (grant number 665779). M. Gallinaro acknowledges the support from the Fundacāo para a Ciência ea Tecnologia (FCT), Portugal (grants IF/00410/2012 and CERN/FISPAR/0006/2017). D. González-Díaz acknowledges the support from MINECO (Spain) under the Ramon y Cajal program (contract RY C-2015-18820). F.J. I guaz acknowledges the support from the Enhanced Eurotal ents program (PCOFUND-GA-2013-600382). S. White acknowledges partial support through the US CMS program under DOE contract No. DE-AC02-07CH11359.

\section{References}

[1] F. Sauli. "Principles of Operation of Multiwire Proportional and Drift Chambers". In CERN Academic Training Lecture no.81, given in the CERN Academic Training Program (May 1977).

[2] T. Papaevangel ou et al. "Fast Timing for High-Rate Environments with Micromegas". In EPJ Web of Conferences, vol ume 174, (2018). 4th International Conference on Micro Pattern Gaseous Detectors (MPGD 2015). 
[3] J. Bortfeldt et al for the RD-51 PICOSEC Collaboration. "PICOSEC: Charged particle timing at sub-25 picosecond precision with a Micromegas based detector". Nucl. Instrum Meth. A, 903:317-325, (2018).

[4] F. J . Iguaz et al. "Charged particle timing at sub-25 pi cosecond precision: the PICOSEC detection concept". 14th Pisa Meeting on Advanced Detectors : Frontier Detectors for Frontier Physics, (2018). accepted to Nucl. Instr. Meth. A.

[5] V. Niaouris. "Picosecond MicroMegas signal processing with Kaiser Bessel Filters". Diploma Thesis, (2017).

[6] S. E. Tzamarias and G. K. Fanourakis. "Analysis Methods and Results of the Picosecond-Micromegas Laser Beam Data, Part A, Charge and Amplitude Properties of the PICOSEC Waveforms". RD51-NOTE-2017009, (2017).

[7] S. E. Tzamarias and G. K. Fanourakis. "Anal ysis Methods and Results of the Picosecond-Micromegas Laser Beam Data, Part B, Timing Characteristics of the PICOSEC Waveforms". RD51-NOTE-2017-010, (2017).

[8] H. Schindler and R. Veenhof. "Garfield++ simulation of tracking detectors". https://garfieldpp.web.cem.ch/garfieldpp/.

[9] K. Paraschou. "Study of the PICOSEC Micromegas Detector with Test Beam Data and Phenomenological Modelling of its Response". Master's thesis, School of Physics, AristotleUniversity of Thessal oniki, (2018).

[10] K. Paraschou, I. Manthos, and S. E. Tzamarias. "Analysis Methods and Results of the PicosecondMicromegas Laser BeamData, Part C, Simulation of the PICOSEC Micromegas and results". RD51-NOTE2017-011, (2017). 